\title{
Impactos ambientales sobre la salud respiratoria de los niños. Carga global de las enfermedades respiratorias pediátricas ligada al ambiente
}

TANIA GAVIDIA*,**, JENNY PRONCZUK*** y PETER D. SLY*,**

Environmental impacts on the respiratory health of children: Global burden of paediatric respiratory diseases linked to the environment

The child's respiratory system is a primary target for air pollutants, including tobacco smoke, biomass fuels and pollution from mobile and fixed sources. Children are, in general more susceptible to the effects of air pollutants; however, individual susceptibility also plays a role. Exposure to pollutants in early life decrease lung growth and results in reduced lung function. The range of respiratory illnesses with an environmental contribution includes: acute viral and bacterial lower respiratory infections; otitis media; asthma and chronic respiratory diseases. This review presents an overview of the identified risks posed by the environment to children's health and their health consequences.

Key words: Air pollutants, environment, children's respiratory diseases.

\section{Resumen}

El sistema respiratorio de los niños es un blanco primario para la contaminación del aire, incluyendo el humo de tabaco, combustibles de biomasa y polución de fuentes móviles y fijas. Los niños son, en general, más susceptibles a los efectos de los contaminantes del aire; sin embargo, la susceptibilidad individual también juega un rol. La exposición a temprana edad reduce el crecimiento pulmonar y resulta en función pulmonar disminuida. El rango de enfermedades respiratorias con una contribución causal ambiental incluye: infecciones agudas virales y bacterianas bajas; otitis media; asma y enfermedades respiratorias crónicas. Esta actualización es una revisión sobre los riesgos ambientales identificados como riesgos para la salud de los niños y sus consecuencias.

Palabras clave: Contaminantes aéreos, ambiente, salud respiratoria infantil.

\section{Glosario de abreviaturas}

CO Monóxido de carbono

DEP Diesel exhaust particles

Partículas de escape Diesel

ETS Environmental tobacco smoke

Humo de tabaco ambiental

$\mathrm{H}_{2} \mathrm{SO}_{4} \quad$ Ácido sulfúrico

HCHO Formaldehido

$\mathrm{NO}_{2} \quad$ Óxido nitroso
PAH Polyaromatic hidrocarbons Hidrocarburos aromáticos policíclicos

PM Particulate matter Material particulado

$\mathrm{PM}_{2,5} \quad$ Material particulado con diámetro $\leq 2,5 \mu \mathrm{m}$

$\mathrm{PM}_{10}$ Material particulado con diámetro $\leq 10 \mu \mathrm{m}$

$\mathrm{SO}_{2} \quad$ Anhídrido sulfuroso o dióxido de azufre

VOCs Volatile organic compounds Compuestos orgánicos volátiles

\footnotetext{
* World Health Organization Collaborating Centre for Research on Children's Environmental Health, School of Public Health, Curtin University of Technology, Perth, Australia.

** Telethon Institute for Child Health Research and Centre for Child Health Research, University of Western Australia.

*** Public Health and Environment, World Health Organization, Ginebra, Suiza.
} 


\section{Introducción}

Un amplio número de exposiciones ambientales han sido relacionadas con enfermedades respiratorias y problema de desarrollo en niños. Tanto en países industrializados como en desarrollo, la mala calidad del aire, tanto intradomiciliario como exterior, aumenta el riesgo de enfermedades respiratorias junto a otros factores como condiciones habitacionales, falta de higiene y conductas no saludables ${ }^{1}$. Mientras las fuentes de contaminación pueden variar en diferentes partes del mundo, el impacto de la exposición a la contaminación ambiental en la salud respiratoria de los niños es una preocupación en todas partes. La Organización Mundial de la Salud (OMS) estima que a nivel mundial hay más de tres mil millones de personas dependientes de combustibles sólidos, incluyendo biomasa (leña, estiércol y residuos agrícolas) para cubrir sus necesidades de energía más básicas: calefacción, hervir agua y cocinar, conducentes a una seria polución intradomiciliaria ${ }^{2}$. En las áreas urbanas los niños pequeños que juegan en las calles y aquellos transportados en cochecillos, respiran a la altura de los tubos de escape por lo que pueden estar expuestos a hidrocarburos no quemados, monóxido de carbono, óxidos de azufre y de nitrógeno y otros tóxicos, que se suman a las concentraciones de material particulado, cenizas y plomo en las áreas donde aun se usa gasolina con plomo. Mientras caminan al colegio a través de áreas de tráfico vehicular denso o en la cercanía de industrias contaminantes los niños pueden exponerse a una miríada de contaminantes dañinos que afectan su función pulmonar, su desarrollo y bienestar ${ }^{3}$.

La contribución estimada de la contaminación del aire a las infecciones respiratorias bajas es, aproximadamente, sobre $40 \%$ en países en desarrollo y $20 \%$ en los industrializados. Por añadidura la influencia de otros factores ambientales como enfriamiento y aglomeración y las comorbilidades parcialmente atribuibles al ambiente (malaria y diarrea por ejemplo) son difíciles de cuantificar, pero pueden sumarse a la carga ambiental facilitadora de infecciones respiratorias bajas ${ }^{4}$.

Los niños son generalmente más vulnerables ante tales exposiciones y la carga de enfermedad pesa desproporcionadamente sobre los niños de países en desarrollo y de bajos ingresos. Los niños menores de 5 años de edad son especialmente vulnerables: hasta un $56 \%$ de todas las muertes atribuibles a contaminación doméstica corresponden a niños de este grupo etáreo ${ }^{5}$.
El impacto de una exposición en particular dependerá, en parte, de la etapa del desarrollo del niño en que ocurre la exposición y su susceptibilidad individual ${ }^{6}$. Esta revisión se centrará en las exposiciones ambientales que tienen un efecto adverso en la salud respiratoria de los niños y en las condiciones respiratorias que causan.

\section{¿Por qué los niños son más susceptibles a exposiciones ambientales?}

El concepto que los niños no son adultos pequeños es ampliamente aceptado por los pediatras. Esta visión es especialmente relevante para comprender la vulnerabilidad de los niños a exposiciones ambientales adversas y ha sido recientemente revisada ${ }^{7}$. Además de la comúnmente reconocida vía de exposición inhalatoria, los niños tienen vías de exposición únicas que incluyen la transplacentaria (in utero), la dérmica y la ingestión no nutricional que aumentan su exposición por efecto de contaminantes del aire que sedimentan. Los niños presentan además un estado anabólico activo lo que significa que respiran más aire, beben más agua e ingieren más comida por unidad corporal que los adultos, lo que significa una mayor dosis ante una exposición ambiental dada. El comportamiento infantil de llevarse constantemente sus manos a la boca conduce a exposición de contaminantes sedimentados a través del contacto con superficies contaminadas y transferencia de los contaminantes a la boca por las manos, juguetes y objetos domésticos.

El sistema respiratorio es particularmente vulnerable a las exposiciones ambientales adversas debido al prolongado período postnatal de crecimiento y desarrollo que presenta ${ }^{8}$. El impacto de exposiciones prenatales vía transplacentaria será discutida más adelante. Muchos órganos están completamente desarrollados al nacer o completan el proceso en el período postnatal temprano ${ }^{8}$. El pulmón está en desarrollo tanto durante la vida pre como postnatal. El patrón de ramificacion de las vía aéreas está completo alrededor de las 16 a 18 semanas de gestación, pero la alveolarización sólo empieza en el tercer trimestre. Al nacimiento el pulmón tiene alrededor del 30 a 50\% de la dotación final de alvéolos del adulto, desarrollándose el resto postnatalmente. La velocidad del crecimiento alveolar es más rápida en los primeros 18 a 24 meses y la microvasculatura pulmonar se desarrolla durante este período de alveolarización. No se sabe cuándo termina el desarrollo alveolar postnatal y las estimaciones, basadas en datos muy limitados, van de 2 a 8 años ${ }^{8}$. 


\section{¿Qué exposiciones ambientales tienen efectos nocivos sobre la salud respiratoria de los niños?}

Las exposiciones ambientales que impactan la salud respiratoria de los niños difieren en los países en desarrollo y en los países desarrollados, como también dentro de un mismo país. Sin embargo, mientras los contaminantes y sus fuentes difieren, existen muchos factores en común. Las exposiciones de los niños ocurren en su hogar, en su vecindario o dentro del ambiente global. Mientras el mayor contribuyente a las infecciones respiratorias agudas bajas, incluidas neumonías, son en países de bajos ingresos los productos emanados de la combustión de biomasa, en las grandes ciudades los efectos respiratorios pediátricos están relacionados principalmente a contaminantes ligados al tráfico vehicular. En los países industrializados la exposición al humo de tabaco ambiental y compuestos orgánicos volátiles dentro de los hogares, como también exposiciones a alergenos, están también entre las principales causas o gatillantes de enfermedad respiratoria ${ }^{9}$.

Tanto las características de los pulmones de los niños como las de los agentes tóxicos individuales influyen sobre la dosis de exposición y órgano afectado. Los niños tienen vías aéreas geométricamente menores que los adultos con un patrón de depósito probablemente más central. Además, el menor tamaño de las vías aéreas del niño condicionaría que los agentes tóxicos tengan un mayor impacto sobre la salud. Como la resistencia de la vía aérea varía inversamente con la cuarta potencia del radio (es decir, resistencia es $1 / \mathrm{radio}^{4}$ ), una reducción de $1 \mathrm{~mm}$ en el radio interno de la vía aérea de un adulto con un diámetro de sección transversal de 20 $\mathrm{mm}$, debido a edema por exposición a agentes tóxicos, significa una reducción de $19 \%$ en el área de sección transversal y un aumento de aproximadamente $50 \%$ de la resistencia. En cambio, la mismo reducción de $1 \mathrm{~mm}$ en una vía aérea infantil con un diámetro de sección transversal de $6 \mathrm{~mm}$ significa un 56\% de reducción en el área de sección transversal y aproximadamente un $500 \%$ de aumento de resistencia.

Las características de los agentes tóxicos también determinan el área del sistema respiratorio, donde se producirán, con mayor probabilidad, los efectos de la exposición. Dos principales características determinan este aspecto: a) cuan soluble en agua es el agente tóxico y b) el tamaño de partícula:

- Agentes tóxicos de alta solubilidad en agua como aldehidos, amoníaco, cloro y $\mathrm{SO}_{2}$ tie- nen mayor probabilidad de afectar los ojos, nariz, faringe y laringe.

- Agentes tóxicos de mediana solubilidad en agua, como el ozono, afectarán las vías aéreas mayores (tráquea y bronquios).

- Agentes tóxicos de baja solubilidad en agua como $\mathrm{NO}_{2}$ afectarán preferentemente a los bronquiolos y alvéolos.

- Partículas grandes sobre $10 \mu \mathrm{m}$ de diámetro se depositan preferentemente en la nariz.

- Partículas gruesas ente 2,5 y $10 \mu \mathrm{m}$ de diámetro probablemente se depositarán en tráquea y bronquios.

- Partículas finas de menos de 2,5 $\mu \mathrm{m}$ de diámetro probablemente se depositarán en bronquiolos y alvéolos.

- Partículas ultrafinas, menores de $1 \mu \mathrm{m}$ de diámetro, pueden ser exhaladas de nuevo por los adultos, pero tienen más probabilidad de depositarse en las vías aéreas más pequeñas de lactantes y niños menores.

\section{Contaminación ambiental}

La exposición a contaminantes aéreos se divide convencionalmente en aquella que tiene lugar en ambientes cerrados y la que ocurre en el aire ambiente global. Esta distinción es algo arbitraria ya que muchos contaminantes se encuentran en las dos situaciones. Sin embargo, esta diferenciación puede emplearse operacionalmente para considerar contaminantes individuales y para examinar sus fuentes más probables.

\section{Contaminación del aire intradomiciliario}

Existen diferencias marcadas entre las fuentes de polución intradomiciliaria en países en vías de desarrollo y en países desarrollados. Una fuente mayor de polución intradomiciliaria en el mundo en desarrollo, especialmente en áreas rurales, es la combustión de biomasa y de carbón para cocinar y calefaccionar ${ }^{2}$. En países desarrollados la mala calidad del aire doméstico está más relacionada con la ventilación inadecuada en casas hechas "herméticas" para reducir gasto en energía, con el humo de tabaco ambiental $\mathrm{y}$ con agentes tóxicos liberados por materiales de construcción.

\section{Humo de tabaco ambiental (ETS)}

El humo de cigarrillo puede ser considerado como constituido por dos fuentes principales: la 
corriente principal que es la inhalada desde el cigarrillo por el fumador y la corriente lateral, liberada directamente al aire ambiental por el cono de combustión del cigarrillo. Estas dos formas de humo difieren en la concentración y naturaleza de componentes químicos, principalmente por sus diferentes condiciones de combustión. La temperaturas máximas en el cono de combustión llegan a $800-900{ }^{\circ} \mathrm{C}$ durante las inhalaciones, mientras que entre inhalaciones la temperatura es sólo de $600{ }^{\circ} \mathrm{C}$, resultando una combustión incompleta del tabaco. La mayor fuente de ETS en los hogares es la corriente lateral.

\section{Material particulado y otros productos relacionados con la combustión}

En ningún área es mayor la disparidad de exposición de los niños de países en desarrollo y de países desarrollados que en la exposición intradomiciliaria a material particulado. En niños que viven en áreas rurales de bajos ingresos de países en desarrollo la exposición a material particulado de combustibles sólidos o biomasa puede ser de varios órdenes de magnitud superior que la de niños de áreas de altos ingresos en países desarrollados.

\section{Fuentes}

- Biomasa y combustibles sólidos: Como ya se dijo la mitad de la población mundial depende de combustibles sólidos (estiércol, leña, residuos agrícolas, carbón, etc.) para sus necesidades básicas de energía. Cocinar y calefaccionar con combustibles sólidos conduce a altos niveles de contaminación intradomiciliaria con una compleja mezcla de contaminantes dañinos para la salud como son el material particulado, monóxido de carbono, $\mathrm{SO}_{2}$, etc. Las principales fuentes son fogones abiertos, estufas y cocinas sin tuberías de eliminación o con escapes de estas, artefactos mal mantenidos, hornos e incineradores cercanos a la casa.

- Otros contaminantes relacionados a combustión. Sus fuentes incluyen ETS, estufas de gas y leña; calefactores a gas, parafina o leña, especialmente los calefactores sin ductos; velas e incienso; espirales antimosquitos; hornos para asados e incineradores cercanos a la casa.

- Espirales antimosquitos. Pueden representar un serio riesgo potencial para la salud de los niños. Su uso prolongado se ha asociado a incidencia aumentada de asma y sibilancias persistentes. Los ingredientes activos son pe- queñas cantidades de piretrinas, consideradas como insecticida de baja toxicidad, pero más del 99\% de la masa de la espiral está constituida por ingredientes llamados "inertes", cuyo humo se ha demostrado que está compuesto de partículas respirables, algunas bastante pequeñas, que contienen hidrocarburos aromáticos policíclicos (PAH) y compuestos de carbonilo, incluyendo formaldehído (HCHO).

Los productos de combustión que pueden contaminar el aire intradomiciliario incluyen:

- Material particulado: $\mathrm{PM}_{10}, \mathrm{PM}_{2,5}$ y partículas ultrafinas.

- Monóxido de carbono (CO): Gas incoloro e inodoro producto de la combustión incompleta de combustibles con carbono.

- $\mathrm{NO}_{2}$ : Formado a altas temperaturas al quemar gas natural y leña en países desarrollados y al quemar leña, parafina o kerosén y biomasa en países en desarrollo. El $\mathrm{NO}_{2}$ es irritante de las mucosas.

- $\mathrm{SO}_{2}$ : Generado especialmente de la combustión de carbón, irrita el epitelio respiratorio.

- Hidrocarburos aromáticos policíclicos, benceno y HCHO: provenientes de biomasa en combustión.

- Metales pesados y fluoruros: Pueden ser emitidos por la combustión de carbón en algunas localidades.

\section{Bioaerosoles}

Los contaminantes biológicos específicos intradomiciliarios incluyen: hongos, ácaros del polvo, caspa de mascotas, animales domésticos y roedores, insectos, agua contaminada, bacterias y virus. La permeación de agua de lluvia o de agua subterránea y el vapor producido por personas, mascotas, cocinas $y$, duchas requieren ventilación adecuada para evitar problemas de humedad que favorecen la proliferación de hongos y microbios. Colchones, fundas de muebles y alfombras son reservorios de ácaros del polvo.

\section{Compuestos orgánicos volátiles}

Los compuestos orgánicos volátiles (sigla en inglés VOC) comprometen significativamente la calidad del aire intradomiciliario. Incluyen múltiples compuestos como hidrocarburos alifáticos, aromáticos y clorados, alcoholes, aldehidos, cetonas, bencenos y formaldehído. Los VOC del ambiente exterior, como los derivados del tráfico, también pueden contaminar el aire intradomiciliario, donde pueden concentrarse a niveles muy superiores a los del exterior. La reparación y pintura de casas puede significar 
un aumento de la exposición a estos compuestos.

Sus fuentes son múltiples: pinturas, removedores de pintura, solventes, preservantes de maderas, pulverizadores de aerosoles, limpiadores y desinfectantes, repelentes de insectos, desodorantes ambientales, combustibles almacenados y productos automotrices, materiales usados en aficiones, ropa limpiada en seco, etc. Los muebles nuevos pueden ser importantes fuentes de VOC's.

\section{El ambiente construido}

Esta denominación de refiere a casas, oficinas y otras estructuras construidas por el hombre en las cuales las personas viven, trabajan o juegan. Los materiales usados en su construcción pueden liberar agentes tóxicos hacia el ambiente y la falta de ventilación e higiene aumentan el riesgo de exposición que puede ser incrementado por la calefacción y acondicionamiento de aire defectuoso.

Fuentes: Materiales de construcción: asbestos, preservantes de madera, pinturas, etc; sistemas de ventilación y acondicionamiento de aire: bacterias, hongos y sus productos; sistemas de calefacción: bacterias, hongos y sus productos.

\section{Contaminación del aire ambiental}

Un problema significativo para la comprensión de la carga de salud ligada a la polución del aire es que las estimaciones de su impacto están mayoritariamente basadas en los resultados de investigaciones realizadas en Europa y Norteamérica, que se han extrapolado a los países en desarrollo. Estas proyecciones originan considerables incertidumbres porque los países en desarrollo difieren de Europa y Norteamérica en la naturaleza de sus contaminantes, las condiciones y magnitud de las exposiciones y el nivel de salud de sus poblaciones.

Las mayores fuentes de contaminantes para el aire ambiental incluyen la utilización de combustibles fósiles (calefacción doméstica, generación de energía, industria, vehículos motorizados, fundiciones, refinerías, incineración, etc); fenómenos naturales (tormentas eléctricas, incendios forestales, tormentas de polvo y erupción de volcanes) y guerras y conflictos ${ }^{10-14}$. Las fuentes posiblemente varían entre las localidades urbanas y rurales.

Material particulado. Las partículas primarias originadas en fuentes de combustión usualmente consisten en un núcleo carbonáceo con elementos químicos como sulfatos, metales e hidrocarburos aromáticos policíclicos adsorbidos a su superficie. Además, se forman partículas secundarias producidas por reacción química de las partículas primarias en la atmósfera con gases fuertemente oxidantes (óxidos de nitrógeno, ozono, óxidos de azufre), llevando a la formación de nitratos y amoníaco.

Partículas de escape Diesel (DEP). Son partículas ultrafinas de menos de $1 \mu \mathrm{m}$ de diámetro que permanecen suspendidas en el aire por períodos prolongados, por lo que son fácilmente inhaladas. Se consideran particularmente tóxicas.

Compuestos de azufre. Incluyen el dióxido de azufre $\left(\mathrm{SO}_{2}\right)$. Ácido sulfúrico $\left(\mathrm{H}_{2} \mathrm{SO}_{4}\right)$ aerosol que se forma por oxidación del $\mathrm{SO}_{2}$ en presencia de humedad, y el ácido sulfhídrico $\left(\mathrm{H}_{2} \mathrm{~S}\right)$. Las industrias que elaboran o usan ácidos pueden también emitir $\mathrm{H}_{2} \mathrm{SO}_{4}$. El $\mathrm{SO}_{2}$ contribuye a la formación de lluvia ácida.

Monóxido de carbono. Formado por combustión incompleta de combustibles que contienen carbón.

Óxidos de nitrógeno. Sus niveles son generalmente menores en el ambiente externo que en el intradomiciliario.

Ozono. Es un importante contaminante en muchas partes del mundo industrializado. Es raramente medido en países en desarrollo de manera que hay poca información de su rol en esos países. La distinción básica que debe tenerse presente es que: a) el ozono "bueno" se encuentra en la alta atmósfera. Es una forma natural del oxígeno que protege a la Tierra de la radiación ultravioleta dañina y b) el ozono "malo" se acumula en la parte baja de la atmósfera. Es un componente mayor de "smog" urbano y un potente irritante que puede aumentar sinergísticamente la reacción de un niño ante otros contaminantes del aire y los pólenes.

El ozono es un contaminante secundario formado por la reacción química entre hidrocarburos y óxidos de nitrógeno en presencia de calor y luz solar. Es importante destacar este último hecho, que explica que el ozono alcance sus máximos niveles en las tardes de verano entre 3 y 5 PM, por lo cual las actividades vigorosas deben planificarse para temprano en la mañana o al oscurecer.

\section{¿Cuáles son los impactos adversos de las exposiciones ambientales sobre la salud de los niños?}

Diversas enfermedades respiratorias pediátricas pueden ser causadas, gatilladas o empeora- 
das por contaminantes en el aire ambiental o intradomiciliario, junto a otros factores como susceptibilidad genética, agentes infecciosos y deficiencias nutricionales e inmunitarias ${ }^{1}$. Las condiciones ligadas a exposiciones ambientales incluyen neumonía, infecciones respiratorias agudas altas y bajas, otitis media aguda, asma y broncoespasmo ${ }^{3}$. Otras condiciones menos comunes son el síndrome de muerte súbita infantil, asociado a ETS; neumonitis aguda hemorrágica, rara condición atribuida a la inhalación de hongos tóxicogénicos que crecen en ambiente húmedo y una amplia gama de síntomas resultantes de exposición en el contexto de trabajo infantil. Además, la exposición prenatal se asocia a efectos adversos en el desarrollo fetal ${ }^{15-18}$ que tienen la posibilidad de traducirse en incrementos de riesgo para enfermedades respiratorias en la infancia y posiblemente más adelante. Los hijos de madres que fuman durante el embarazo tienen mayor probabilidad de presentar una función pulmonar reducida ${ }^{19}$ y un mayor riesgo de asma a mayor edad ${ }^{20}$.

\section{Infecciones respiratoria agudas bajas y neumonía}

Globalmente, la neumonía e infecciones respiratorias bajas representan la causa más importante de muerte en niños menores de 5 años. Según el Grupo de Referencia sobre Epidemiología de la Salud del Niño, las principales causas responsables del $73 \%$ de cerca de 11 millones de muertes de niños bajo los 5 años en el bienio 2002 y 2003 fueron: neumonía 19\%; enfermedades diarreicas $18 \%$; malaria $8 \%$; neumonía y sepsis neonatal $10 \%$; parto prematuro $10 \%$ y asfixia al nacer $8 \%$. La desnutrición fue una causa subyacente en el $53 \%$ de estas muertes ${ }^{21}$. Globalmente la neumonía mata más niños que cualquiera otra enfermedad y supera al Sida, a la malaria y al sarampión combinados.

La exposición a contaminación intradomiciliaria más que duplica el riesgo de neumonía. El 36\% de infecciones respiratorias bajas a nivel mundial son atribuibles a los combustibles sóli$\operatorname{dos}^{9,23}$ y el $1 \%$ de todas las infecciones respiratorias, a la contaminación atmosférica ${ }^{9,21}$. En los países industrializados, donde el uso de combustibles sólidos para cocinar y calefaccionar es menos frecuente, son otros contaminantes, especialmente el humo de tabaco, los que juegan el rol más relevante. Un estudio realizado en Italia estima que el $21 \%$ de las infecciones respiratorias agudas de los primeros dos años de vida son debidas al hábito de fumar de los padres. En el Reino Unido entre el 40 y $60 \%$ de los niños están expuestos a humo ambiental de tabaco y existe evidencia sustancial que esto aumenta su riesgo de enfermedad respiratoria baja $\mathrm{a}^{24}$.

\section{Otitis media e infecciones respiratorias altas}

La otitis media o infección del oído medio, es una de las infecciones más comunes en niños pequeños y un motivo muy frecuente de consulta médica en la mayor parte del mundo. Sin embargo, la asociación entre ella y las exposiciones ambientales es poco conocida. La inflamación del oído medio se produce frecuentemente por extensión de infecciones, comúnmente virales, causantes de angina faríngea, resfríos y otros problemas respiratorios. Aproximadamente el $75 \%$ de los niños experimentan al menos un episodio de otitis media antes de su tercer cumpleaños y la mitad de ellos presentarán 3 ó más episodios durante sus primeros tres años de vida. La otitis media es una de las principales razones para prescripción de antibióticos o realización de procedimientos quirúrgicos en los países industrializados ${ }^{25}$. El costo directo e indirecto estimado de esta situación llega a 5.000 millones de dólares al año en Estados Unidos ${ }^{26}$.

La otitis media con derrame puede tener serias consecuencias como pérdida de audición, retraso en el desarrollo del lenguaje y aprendizaje y, si es recurrente, puede reducir la calidad de vida $^{27}$. Estudios recientes de cohortes sugieren una asociación positiva de la otitis, no sólo con el humo de tabaco de segunda mano, sino que también con contaminantes del tráfico como dióxido de nitrógeno, partículas finas $\left(\mathrm{PM}_{2,5}\right)$ y carbono elemental ${ }^{28}$. La contribución ambiental a las infecciones respiratorias altas y otitis medias es estimada en $24 \%$ en países en desarro1lo. Sin embargo, cabe advertir que esta relación está insuficientemente documentada. Como sucede con las infecciones respiratorias bajas, se ha estimado que la incidencia de infecciones respiratorias altas es menor en países industrializados $(12 \%)$.

\section{Asma}

El asma es esencialmente una enfermedad del desarrollo, en la cual el desarrollo normal de los sistemas respiratorio e inmunitario es alterado por los impactos de exposiciones ambientales actuando sobre predisposiciones genéticas subyacentes. La contaminación del aire, tanto exterior como intradomiciliario, ha sido identificada como un factor potencial de riesgo para su iniciación, inducción y exacerbación. Un probable mecanismo es la inducción de inflamación pul- 
monar que afectaría el crecimiento pulmonar post-natal. Los contaminantes que pueden inducir esta inflamación incluyen los productos de la combustión de combustibles orgánicos, material particulado, productos de escape Diesel, bioaerosoles portadores de hongos, alergenos y productos bacterianos (como lipopolisacáridos), y tóxicos como formaldehído y otros compuestos orgánicos volátiles.

Aunque no existe información definitiva, se estima que las infecciones virales repetidas alteran el crecimiento pulmonar, pero sí se ha demostrado que son un factor mayor de riesgo de desarrollo de asma ${ }^{29-34}$.

El impacto de la exposición ambiental a humo de tabaco sobre el desarrollo del asma es un tanto controversial. Como se ha dicho anteriormente, fumar durante el embarazo se asocia una menor función pulmonar al nacer ${ }^{19,35}$ y es un factor mayor de riesgo para sibilancias infanti$\operatorname{les}^{33}$. La relación entre exposición post-natal y crecimiento pulmonar y asma infantil es menos clara. Es difícil separar los efectos de la exposición a ETS pre y post-natal ${ }^{36}$ ya que las mujeres fumadoras con frecuencia no se abstienen en ninguno de los dos períodos. Los estudios hechos en países donde es común que fumen los hombres, pero raro que lo hagan las mujeres demuestran efectos adversos sobre función pulmonar de la exposición post-natal al humo del padre $^{36}$. Un estudio en 360 niños turcos demostró que esta exposición se asocia a la reducción de varios índices de función pulmonar cuando los niños alcanzaban la edad de 9 a 13 años ${ }^{37}$.

Estudios longitudinales también han demostrado el efecto de la contaminación ambiental sobre el crecimiento pulmonar en niños. El Estudio de Salud Infantil en California del Sur reclutó 1.759 niños de edad promedio de 10 años de colegios primarios de 12 comunida$\operatorname{des}^{38}$. Las mediciones de contaminación demostraron concomitancias significativas entre el $\mathrm{NO}_{2}$, vapor ácido, y material particulado entre todas las 12 comunidades, demostrando que estos contaminantes actuaban en conjunto como un "paquete". El ozono no se correlacionaba con los otros contaminantes. La función pulmonar fue medida secuencialmente durante 8 años y mostró un crecimiento lento. Este afectó especialmente el $\mathrm{VEF}_{1}$ cuyo déficit se asoció con exposición a $\mathrm{NO}_{2}(\mathrm{p}=0,005)$, vapor ácido $(\mathrm{p}=0,004), \mathrm{PM}_{2,5}$ $(p=0,04)$ y carbón elemental $(p=0,007)$.

Un metanálisis reciente revisó los estudios publicados entre 1970 y 2005 sobre el efecto de ETS y desarrollo de asma en niños ${ }^{39}$. Entre 300 artículos potencialmente relevantes analizó 38 estudios epidemiológicos que cumplieron los criterios de inclusión y comunicó un riesgo relativo conjunto de 1,33 (95\% CI: 1,14-1,56) para niños entre 6 y 16 años de edad, habiéndose controlado la historia atópica familiar y personal y el consumo de cigarrillo de los niños. Esta estimación era 1,27 veces mayor que la calculada para niños menores y los autores concluyen que la duración de la exposición a ETS es un factor importante en la inducción de asma. Similarmente, Kulkarni et $\mathrm{al}^{40}$, estudiaron los macrófagos obtenidos de la expectoración inducida de niños entre 5 y 15 años de edad y midieron la cantidad de carbón elemental dentro de los macrófagos como índice de exposición. Comunicaron una inferior función pulmonar en los niños más intensamente expuestos con una reducción de $17 \%$ en el $\mathrm{VEF}_{1}$, de $12,9 \%$ en la CVF y de $34,7 \%$ en el $\mathrm{FEF}_{25-75}$ por cada $1 \mu \mathrm{m}^{2}$ de aumento del área ocupada por carbón en los macrófagos ${ }^{40}$.

\section{Susceptibilidad genética y efectos epigenéticos}

Los mayores efectos sobre la salud de muchos tóxicos ambientales involucran la inducción de inflamación y estrés oxidativo. Los efectos combinados de estos procesos pueden causar daño agudo o crónico a los pulmones. El sistema respiratorio tiene un sistema de defensa antioxidante que incluye enzimas metabólicas de fase II como la superóxido dismutasa y la familia de la glutation-S-transferasa, que detoxifican los productos de la inflamación potencialmente dañinos. Se han descrito variantes genéticas de estas enzimas que aumentan el riesgo para la salud de agentes tóxicos inhalados.

Los sistemas respiratorio e inmunitario son particularmente vulnerables a la interacción gene/ ambiental que se considera que juega un rol sustancial en el desarrollo de asma y alergias. Esto se debe, en parte, al prolongado período de maduración de estos sistemas tanto en el desarrollo tanto pre como post-natal. También están involucradas muchas vías complejas en el desarrollo de estos sistemas.

La predisposición genética es reconocida como un importante componente del riesgo de desarrollar una sensibilización alérgica, como se manifiesta en familias con historia de alergias. Los niños de éstas muestran una maduración inmunitaria post-natal arrastrada, evidenciada por una reducida proliferación de células $\mathrm{T}$ ante estimulación "in vitro" y una disminuida capacidad de secretar citoquinas, especialmente Th-1, todo lo cual es un factor de riesgo mayor para 
el desarrollo de sensibilización y asma atópica más tarde en la vida.

Existen muchas áreas potenciales donde las interacciones gene-ambientales pueden jugar un rol en el desarrollo de alergias y asma:

- El impacto del fumar materno durante el embarazo sobre la maduración del sistema inmune en la infancia se observa especialmente en niños con más genotipos "proinflamatorios", en genes comunes relacionados con asma y alergias

- El impacto de la exposición a ETS sobre el riesgo de asma es mayor en las mismas circunstancias.

- El impacto de la exposición a contaminación del aire, especialmente por tráfico, sobre el riesgo de desarrollar asma y sobre su severidad es mayor en niños con genotipos con menor capacidad de neutralizar el estrés oxidativo.

El término epigenético se usa para describir los procesos en que la expresión genética es regulada por mecanismos no genéticos. Estos procesos son parte esencial de la organogénesis y maduración donde los factores no genéticos regulan la cronología de la expresión genética. Los mecanismos epigenéticos participan en la supresión de mutaciones potencialmente dañinas, pueden modificar la expresión genética en respuesta a exposiciones ambientales y están involucrados en la expresión transgeneracional de enfermedades. Ejemplos de fenómenos epigenéticos en el aumento del riesgo de asma y alergias incluyen los siguientes:

- El hábito tabáquico materno durante el embarazo también aumenta el riesgo de asma en el nieto (a) de madres expuestas al hábito tabáquico materno durante el embarazo, a través de mecanismos epigenéticos.

- Si la abuela maternal fumó durante el embarazo de una hija, aunque ésta no fume el riesgo de asma de su hijo es un $80 \%$ mayor 1,8 (95\% CI: 1,0-3,3). El riesgo aumenta a $2,6(95 \%$ CI: $1,6-4,5)$ si la madre también fuma durante su embarazo ${ }^{41}$. Esto puede ser explicado por un efecto del tabaco fumado por la abuela sobre el desarrollo in útero de los óvulos del feto femenino y constituyen un ejemplo de un fenómeno epigenético que aumenta el riesgo de asma.

- El estrés psicológico materno aumenta los niveles de $\operatorname{IgE}$ en el cordón umbilical. Este efecto ha sido observado en madres que en tests psicológicos revelan nerviosimo, agotamiento, ansiedad, estrés laboral y depresión, pero no en madres que demuestran vitalidad, vigor y alegría. Es posible que este fenómeno se relacione con supresión de la producción de citoquinas Th- 1 por los trofoblastos de la placenta y con el desarrollo del sistema inmune.

- Asma materna: sus efectos sobre el feto en desarrollo dependen del sexo de éste. Los fetos femeninos son propensos a depresión del eje hipofiso-tálamo adrenal con reducción de crecimiento, menor función adrenal y metabolismo glucocorticoideo placentario reducido. Estos efectos son marcadamente prevenidos con el uso de corticoides inhalados. En contraste los fetos masculinos no presentan dichas alteraciones. Sin embargo, ante un segundo factor estresante como una exacerbación del asma materna el feto masculino está en riesgo de una desaceleración aguda del crecimiento y muerte súbita, efectos que no son evitados con el uso materno de corticoides inhalados ${ }^{43-45}$.

La literatura en esta área se está expandiendo rápidamente de manera que es conveniente que los lectores interesados consulten las publicaciones más recientes para contar con ejemplos actualizados de interacciones gene/ambiente y fenómenos epigenéticos.

\section{Bibliografía}

1.- WHO, Effects of Air Pollution on Children's Health and Development: A review of the evidence. World Health Organization Special Programme on health and Environmnet. European Centre for Environment and Health 2005.

2.- REHFUESS E. Fuel for life: Household energy and Health. 2006, World Health Organization: Geneva.

3.- ETZEL R A, BALAKRISHNAN K. Chapter 10: Air, in Children's health and the environment - A Global Perspective: A Resource Manual for the Health Sector, J. P. d. Garbino, Editor. 2004, WHO: Geneva. p. 107-132.

4.- PRÜSS-ÜSTÜN A, CORVALÁN C. Preventing disease through healthy environments: Towards an estimate of the environmental burden of disease. 2006, World Health Organization: Geneva.

5.- WHO, Fact sheet $\mathrm{N}^{\circ} 292$ : Indoor air pollution and health: Scope of the problem. 2005.

6.- PRONCZUK J, BHAVE S Y. Children's health and the environment - building capacities of action. Indian pediatrics 2007; 44: 253-5.

7.- SLY P D, FLACK F. Susceptibility of children to environmental pollutants. Ann N Y Acad Sci 2008; 
1140: $163-83$.

8.- WHO. Environmental Health Criteria 237: Principles for Evaluating Health Risks in Children Associated with Exposure to Chemicals EHC 2006 [cited 2009 13 January].

9.- WHO, The World Health Report 2002: Reducing Risks, Promoting Healthy Life. 2002, World Health Organization: Geneva.

10.- TENNESSEE M. Chapter 5: Where the child works, in Children's health and the environment - A Global Perspective: A Resource Manual for the Health Sector J.P.d. Garbino, Editor. 2005, WHO: Geneva. p. 46-53.

11.- PRONCZUK J. Chapter 4: Where the child learns, in Children's health and the environment- A Global Perspective: A Resource Manual for the Health Sector, J.P.d. Garbino, Editor. 2005, WHO: Geneva. p. 40-45.

12.- CHAUDHURI N, FRUCHTENGARTEN L. Chapter 3: Where the child lives and plays, in Children's health and the environment - A Global Perspective: A Resource Manual for the Health Sector, J.P.d. Garbino, Editor. 2005. p. 29-39.

13.- BU-HAKAH R. Chapter 6: Where the child is under extreme stress, in Children's health and the environment - A Global Perspective: A Resource Manual for the Health Sector, J.P.d. Garbino, Editor. 2005, WHO: Geneva. p. 54-63.

14.- WHO/EURO, Air Quality Guidelines Global Update 2005, in WHO Regional Office for Europe. 2006, WHO Copenhagen $\varnothing$, Denmark.

15.- LIU S, KREWSKI D, SHI Y, CHEN Y, BURNETT R T. Association between maternal exposure to ambient air pollutants during pregnancy and fetal growth restriction. J Expo Sci Environ Epidemiol 2007; 17: 426-32.

16.- ROGERS J F, DUNLOP A L. Air pollution and very low birth weight infants: a target population? Pediatrics 2006; 118: 156-64.

17.- SRAM R J, BINKOVÁ D, DEJMEK J, BOBAK M. Ambient air pollution and pregnancy outcomes: a review of the literature. Environ Health Perspect 2005; 113: 375-82.

18.- HANSEN C A, BARNETT A G, PRITCHARD G. The effect of ambient air pollution during early pregnancy on fetal ultrasonic measurements during mid-pregnancy. Environ Health Perspect 2008; 116: 362-9.

19.- STICK S M, BURTON P R, GURRIN L, SLY P D, Le SOUËF P N. Effects of maternal smoking during pregnancy and a family history of asthma on respiratory function in newborn infants. Lancet 1996; 348 (9034): 1060-4.

20.- MACAUBAS C, DE KLERK N H HOLT B J, WEE C, KENDALL G, FIRTH M, et al. Association between antenatal cytokine production and the development of atopy and asthma at age 6 years. Lancet 2003; 362 (9391): 1192-7.
21.- BRYCE J, BOSCHI-PINTO C, SHIBUYA K, BLACK R E, WHO Child Health Epidemiology Reference Group. WHO estimates of the causes of death in children. Lancet 2005; 365 (9465): 1147-52.

22.- UNICEF. Pneumonia: the forgotten killer of children. 2006, UNICEF? WHO: Geneva.

23.- SMITH K R, MEHTA S. The burden of disease from indoor air pollution in developing countries: comparison of estimates. Int J Hyg Environ Health 2003; 206: 279-89.

24.- RUSHTON L. Health impact of environmental tobacco smoke in the home. Rev Environ Health 2004; 19: 291-309.

25.- ROVERS M M, SCHILDER A G, ZIELHUIS G A, ROSENFELD R M. Otitis media. Lancet 2004; 363 (9407): 465-73.

26.- GATES G A. Cost-effectiveness considerations in otitis media treatment. Otolaryngol Head Neck Surg 1996; 114: 525-30.

27.- BROUWER C N, MAILLÉ A R, ROVERS M M GROBBEE D E, SANDERS E A, SCHILDER A G. Health-related quality of life in children with otitis media. Int J Pediatr Otorhinolaryngol 2005; 69: 103141.

28.- BRAUER M, GEHRING U, BRUNEKREEF B, DE JONGSTE J, GERRITSEN J, ROVERS M, et al. Trafficrelated air pollution and otitis media. Environ Health Perspect 2006; 114: 1414-8.

29.- ODDY W H, DE KLERK N H, SLY P D, HOLT P G. The effects of respiratory infections, atopy, and breastfeeding on childhood asthma. Eur Respir J 2002; 19: 899-905.

30.- ODDY W H, PEAT J K, DE KLERK N H. Maternal asthma, infant feeding, and the risk of asthma in childhood. J Allergy Clin Immunol 2002; 110: 65-7.

31.- ODDY W H, SLY P D, DE KLERK N H, LANDAU L I, KENDALL G E, HOLT P G, et al. Breast feeding and respiratory morbidity in infancy: a birth cohort study. Arch Dis Child 2003; 88: 224-8.

32.- WRIGHT A L, TAUSSIG G L, RAY C G, HARRISON H R, HOLBERG C J. The Tucson Children's Respiratory Study. II. Lower respiratory tract illness in the first year of life. Am J Epidemiol 1989; 129: 1232-46.

33.- MARTÍNEZ F D, WRIGHT A L, TAUSSIG L M, HOLBERG C J, HALONEN M, MORGAN W J. Asthma and wheezing in the first six years of life. The Group Health Medical Associates. N Engl J Med 1995; 332: 133-8.

34.- KUSEL M M, DE KLERK N H, HOLT P G, KEBADZE T, JOHNSTON S L, SLY P D. Role of Respiratory Viruses in Acute Upper and Lower Respiratory Tract Illness in the First Year of Life: A Birth Cohort Study. The Pediatric Infectious Disease Journal 2006; 25: 680-6.

35.- LODRUP CARLSEN K C, JAAKKOLA J J, NAFSTAD 
$\mathrm{P}$, CARLSEN K H. In utero exposure to cigarette smoking influences lung function at birth. Eur Respir J 1997; 10: 1774-9.

36.- LE SOUEF P N. Pediatric origins of adult lung diseases. 4. Tobacco related lung diseases begin in childhood. Thorax 2000 55: 1063-7.

37.- BEK K, TOMAÇ N, DELIBAS A, TUNA F, TEZIÇ H T, SUNGUE M. The effect of passive smoking on pulmonary function during childhood. Postgrad Med J 1999; 75 (884): 339-41.

38.- GAUDERMAN W J, AVOL E, GILLILAND F, VARA $\mathrm{H}$, THOMAS D, BERHANE $\mathrm{K}$, et al. The effect of air pollution on lung development from 10 to 18 years of age. N Engl J Med 2004; 351 (11): 1057-67.

39.- VORK K L, BROADWIN R L, BLAISDELL R J. Developing asthma in childhood from exposure to secondhand tobacco smoke: insights from a metaregression. Environ Health Perspect 2007; 115: 1394400.

40.- KULKARNI N, PIERSE N, RUSHTON L, GRIGG J. Carbon in airway macrophages and lung function in children. N Engl J Med 2006; 355: 21-30.

41.- LI Y F, LANGHOLZ B, SALAM M T, GILLILAND F
D. Maternal and grandmaternal smoking patterns are associated with early childhood asthma. Chest 2005; 127: 1232-41.

42.- LIN Y C, WEN H J, LEE Y L, GUO Y L. Are maternal psychosocial factors associated with cord immunoglobulin $\mathrm{E}$ in addition to family atopic history and mother immunoglobulin E? Clin Exp Allergy 2004; 34: 548-54.

43.- CLIFTON V. Maternal asthma during pregnancy and fetal outcomes: potential mechanisms and possible solutions. Curr Opin Allergy Clin Immunol 2006; 6: 307-11.

44.- CLIFTON V L, MURPHY V E. Maternal asthma as a model for examining fetal sex-specific effects on maternal physiology and placental mechanisms that regulate human fetal growth. Placenta 2004; 25 Suppl A: S45-52.

45.- CLIFTON V L, RENNIE N, MURPHY V E. Effect of inhaled glucocorticoid treatment on placental 11 betahydroxysteroid dehydrogenase type 2 activity and neonatal birthweight in pregnancies complicated by asthma. Aust N Z J Obstet Gynaecol 2006; 46: 13640 .

\section{Correspondencia a:}

Dr. Peter D. Sly

World Health Organization Collaborating Centre for Research on Children's Environmental Health, School of Public Health, Curtin University of Technology, Perth, Australia.

E-mail: peters@ichr.uwa.edu-au 